\title{
Improved Bounds for the Transition Temperature of Directed Polymers in a Finite-Dimensional Random Medium
}

\author{
M. R. Evans ${ }^{1}$ and B. Derrida ${ }^{1}$
}

Received March 20, 1992

\begin{abstract}
We consider the problem of directed polymers in a random medium of a finitedimensional lattice. In the high-temperature phase of this system it is known that the annealed and quenched free energies coincide. Upper bounds on the transition temperature to a low-temperature phase had previously been obtained by calculating the first two moments $\langle Z\rangle$ and $\left\langle Z^{2}\right\rangle$ of the partition function. We improve these bounds by estimating noninteger moments $\left\langle Z^{\alpha}\right\rangle$ for $1<\alpha<2$.
\end{abstract}

KEY WORDS: Disorder; random systems; directed polymer; noninteger moments of the partition function; bounds.

\section{INTRODUCTION}

From the study of strongly disordered systems such as spin glasses carried out in the last decade ${ }^{(1)}$ several particular features of the low-temperature phase have emerged. At least in the mean-field limit the low temperature phase is characterized by a phase space randomly broken into free energy valleys, the weights of which vary from sample to sample and remain non-self-averaging even in the limit of infinitely large systems. ${ }^{(1,2)}$ Attempts to generalize these ideas to finite-dimensional systems ${ }^{(3)}$ have proven rather difficult and conflicting opinions remain as to whether the mean-field results provide the right picture. Even demonstrating that spin glasses do have a phase transition in finite dimension is not an easy task.

Recently it has become apparent that the problem of directed polymers in a random medium ${ }^{(4,5)}$ has many features in common with that

\footnotetext{
${ }^{1}$ Service de Physique Théorique (Laboratoire de la Direction des Sciences de la Matière du Commissariat à l'Energie Atomique), C.E. Saclay, F-91191 Gif-sur-Yvette Cedex, France.
} 
of spin glasses. In particular, the mean-field limit, ${ }^{(6)}$ which is realized by considering directed polymers on a tree, has a low-temperature phase with analogous properties to that of the spin-glass phase of the SherringtonKirkpatrick model as described by Parisi's approach. However, while yielding this behavior, the directed polymer problem is simple enough to allow, in the mean-field limit, for a closed expression of the free energy at all temperatures. Together with the close relationship of directed polymers to growth models, ${ }^{(7)}$ this provides a motivation to study directed polymers in finite dimension.

A key difference that appears in the finite-dimensional model is that two polymers can rejoin once they have parted on a finite-dimensional lattice, whereas on a tree they cannot. This feature was systematically introduced to obtain a $1 / d$ expansion in high enough dimension. ${ }^{(8)}$ The existence of a phase transition in finite dimension was also proven by a very simple argument based on the calculation of the first two moments of the partition function $Z$. This argument allows one to obtain bounds on the free energy and transition temperature $T_{c} \cdot{ }^{(9,10)}$ When the argument is used in the mean-field theory the upper bound on $T_{c}$ is strictly higher than the known $T_{c}$ and so one gets the feeling that the bounds could be improved upon. This suspicion has recently been confirmed by an approach based on martingales that was developed to estimate the noninteger moments $\left\langle Z^{\alpha}\right\rangle$ of $Z$ for $1<\alpha \leqslant 2$ in the mean-field case ${ }^{(11)}$ (see also refs. 12 and 13 for the application of martingales to tree problems). It was shown that the bounds on $T_{c}$ could be improved to the extent that the exact $T_{c}$ was recovered, thus providing a new route to the exact solution of the model.

The purpose of the present paper is to follow a similar approach based on the estimate of noninteger moments, but in the finite-dimensional case, to obtain improved bounds on the transition temperature. The paper is organized as follows: Section 2 recalls the problem of directed polymers and how integer moments may be used to obtain bounds on the transition temperature; in Section 3 we show how these ideas may be generalized to noninteger moments, for which we obtain inequalities; in Section 4 numerical values for the upper bound on the transition temperature are extracted from the inequalities derived in Section 3 and possible improvements are discussed.

\section{INTEGER MOMENTS OF THE PARTITION FUNCTION FOR DIRECTED POLYMERS}

Directed polymers are directed random walks of length $L$ through a lattice with fixed origin. Each site $\mathbf{r}$ of the lattice has a random variable $\varepsilon_{\mathbf{r}}$, 
known as a site energy, associated with it and we use the convention that the energy $\varepsilon_{\mathbf{o}}$ of the origin $\mathbf{o}$ is zero. The energy of a walk $W$ is the sum of the energies at the sites through which the walk passes:

$$
E_{W}=\sum_{\mathbf{r} \in W} \varepsilon_{\mathbf{r}}
$$

The partition function for polymers of length $L$ may then be defined as

$$
Z_{L}=\sum_{W} e^{-\beta E_{W}}
$$

where $\beta$ is the inverse temperature. In this work we consider walks directed in the $(1,1, \ldots, 1)$ direction of a hypercubic lattice, which means that at each step of the walk one coordinate increases by one. There are thus $d^{L}$ possible walks, where $d$ is the dimension of the lattice.

Let us first recall how from the knowledge of the first two moments $\langle Z\rangle$ and $\left\langle Z^{2}\right\rangle$ one can obtain the expression of the quenched free energy at high enough temperature. Consider the ratio $R_{2}$ defined by

$$
R_{2}(\beta)=\lim _{L \rightarrow \infty} \frac{\left\langle Z_{L}^{2}(\beta)\right\rangle}{\left\langle Z_{L}(\beta)\right\rangle^{2}}
$$

If $R_{2}$ is finite, then it can be shown by a simple application of Chebyshev's inequality that

$$
\lim _{L \rightarrow \infty} \frac{\log Z_{L}}{L}=\lim _{L \rightarrow \infty} \frac{\log \left\langle Z_{L}\right\rangle}{L}
$$

holds with probability greater than $1 / R_{2}$. To calculate $R_{2}$, we need to know the average of the partition function, which is simply the number of walks multiplied by the average weight of a walk:

$$
\left\langle Z_{L}\right\rangle=d^{L}\left\langle e^{-\beta \varepsilon}\right\rangle^{L}
$$

The angular brackets denote an average over the distribution of site energies $\varepsilon$. The quantity $\left\langle Z_{L}^{2}\right\rangle$ may be calculated by noting that $Z_{L}^{2}$ is the partition function of a system whose configurations are pairs of walks. The average weight of a pair of walks depends on how many times the walks meet. One then finds ${ }^{(10)}$

$$
R_{2}=\sum_{m=0}^{L} P_{m}\left(\frac{\left\langle e^{-2 \beta \varepsilon}\right\rangle}{\left\langle e^{-\beta \varepsilon}\right\rangle^{2}}\right)^{m}
$$

where $P_{m}$ is the probability that two walks meet $m$ times. 
The probability of (4) may be increased by considering polymers with the energy of the first few steps removed. This process does not affect the extensive part of the free energy, because the first few steps give a nonextensive contribution; however, it does increase the lower bound on the probability that (4) is true. In this way one can remove enough steps when $R_{2}$ is finite to show that (4) holds with a probability tending to one. ${ }^{(10)}$ Thus, when $R_{2}$ is finite, the free energy per monomer is given by the annealed free energy $\lim _{L \rightarrow \infty} \log \left\langle Z_{L}\right\rangle / L$ with probability one.

In high enough dimension $(d>3) P_{m}$ decays exponentially with $m$ for $L \rightarrow \infty$,

$$
P_{m}=(1-A) A^{m}
$$

The constant $A$, which is lattice dependent, is the probability that two directed walks starting from the origin subsequently meet at least once, and can be computed explicitly. ${ }^{(10)}$ By inserting (7) into (6), one sees that the condition for $R_{2}$ to be finite is that $T>T_{2}$, where $T_{2}$ is the solution of

$$
\frac{\left\langle e^{-2 \beta \varepsilon}\right\rangle}{\left\langle e^{-\beta \varepsilon}\right\rangle^{2}} A=1 \quad \text { with } \quad \beta=\left[T_{2}\right]^{-1}
$$

So for $T>T_{2}$, the quenched free energy is equal to the annealed free energy, whereas for $T<T_{2}$ the above argument does not tell us whether the annealed free energy is still equal to the quenched free energy or not. On the other hand, a necessary condition on the annealed free energy for it to be equal to the quenched free energy is that it does not give a negative entropy. The condition may be written as

$$
\frac{\partial}{\partial \beta}\left\langle Z_{L}(\beta)\right\rangle^{1 / \beta}<0
$$

The temperature at which $(9)$ is violated then gives a lower bound $T_{0}$ below which the annealed and quenched free energies must be different. ${ }^{(10)}$ So a phase transition has to occur at a temperature $T_{c}$ which lies between $T_{0}$ and $T_{2}$ :

$$
T_{0} \leqslant T_{c} \leqslant T_{2}
$$

In this work we will consider a Gaussian distribution of site energies with unit variance. This results in

$$
\langle\exp (-\beta \varepsilon)\rangle=\exp \left(\beta^{2} / 2\right)
$$

For a tree of branching number $d, A$ in (7) is the probability that two walks follow the same branch at the first step, so that $A=1 / d$. The upper 
and lower bounds given by (8) and (9) for a tree and Gaussian energies are then

$$
T_{0}=\frac{1}{(2 \log d)^{1 / 2}} \leqslant T_{c} \leqslant \frac{1}{(\log d)^{1 / 2}}=T_{2}
$$

We know from other approaches ${ }^{(6,11,12)}$ that the lower bound $T_{0}$ is in fact the correct transition temperature $T_{c}$. Evidently there is room for improvement in the upper bound.

\section{NONINTEGER MOMENTS}

Our discussion of the noninteger moments of the partition function will center on the ratio

$$
R_{\alpha}=\lim _{L \rightarrow \infty} \frac{\left\langle Z_{L}^{\alpha}(\beta)\right\rangle}{\left\langle Z_{L}(\beta)\right\rangle^{\alpha}} \quad \text { where } \quad 1<\alpha \leqslant 2
$$

The trouble with dealing with noninteger moments is that closed expressions similar to (6) can no longer be obtained. Instead, we shall obtain bounds on the ratio $R_{\alpha}$. Our main tools will be two well-known inequalities ${ }^{(14)}$ : the convexity inequality (due to Jensen), which states that for $x \geqslant 0$

$$
\begin{array}{ll}
\left\langle x^{\gamma}\right\rangle \leqslant\langle x\rangle^{\gamma} & \text { for } \quad \gamma \leqslant 1 \\
\left\langle x^{\gamma}\right\rangle \geqslant\langle x\rangle^{\gamma} & \text { for } \quad \gamma \geqslant 1
\end{array}
$$

and a standard inequality which states that for any set of $x_{i} \geqslant 0$

$$
\begin{array}{ll}
\left(\sum_{i} x_{i}\right)^{\gamma} \geqslant \sum_{i}\left(x_{i}\right)^{\gamma} \quad \text { for } \quad \gamma \geqslant 1 \\
\left(\sum_{i} x_{i}\right)^{\gamma} \leqslant \sum_{i}\left(x_{i}\right)^{\gamma} \quad \text { for } \quad \gamma \leqslant 1
\end{array}
$$

An immediate consequence for $R_{\alpha}$ of convexity (14) is that

$$
R_{\alpha} \geqslant 1 \quad \text { if } \quad \alpha \geqslant 1
$$

By an analogous argument to that outlined in the previous section for $R_{2}$, it can be shown that if $R_{\alpha}$ is finite, then the annealed is equal to the quenched free energy. For $2 \geqslant \alpha>1$, the temperature $T_{\alpha}$ above which this occurs is less than or equal to $T_{2}$ as can be seen by noting that

$$
R_{\alpha} \leqslant R_{\alpha}^{2 / \alpha}=\lim _{L \rightarrow \infty} \frac{\left\langle Z_{L}^{\alpha}(\beta)\right\rangle^{2 / \alpha}}{\left\langle Z_{L}(\beta)\right\rangle^{2}} \leqslant R_{2}
$$


where convexity (14) has been invoked in the final inequality. $T_{\alpha}$ can then give an improved upper bound on the transition temperature $T_{c}$,

$$
T_{c} \leqslant T_{\alpha} \leqslant T_{2} \quad \text { for } \quad 1<\alpha \leqslant 2
$$

In order to estimate $R_{\alpha}$, we write $Z_{L}^{2}$ as the sum of the partition functions $Y_{L}\left(\{\mathbf{r}\}_{m}\right)$ for pairs of paths that meet at the sites $\{\mathbf{r}\}_{m}=$ $\left\{\mathbf{r}_{0}, \mathbf{r}_{1}, \ldots, \mathbf{r}_{m}\right\}$. The sum is over $m$, the number of times the two paths meet subsequent to their initial intersection $\mathbf{r}_{0}=\mathbf{0}$, and over all possible choices of the $m$ meeting sites $\{\mathbf{r}\}_{m}$ :

$$
\left[Z_{L}\right]^{\alpha}=\left[\left(Z_{L}\right)^{2}\right]^{\alpha / 2}=\left[\sum_{m=0}^{L} \sum_{\{\mathbf{r}\}_{m}} Y_{L}\left(\{\mathbf{r}\}_{m}\right)\right]^{\alpha / 2}
$$

We now make use of inequality (15) for $\alpha / 2 \leqslant 1$ to separate the contributions from different sets of intersections

$$
\left[Z_{L}\right]^{\alpha} \leqslant \sum_{m=0}^{L} \sum_{\{\mathbf{r}\}_{m}}\left[Y_{L}\left(\{\mathbf{r}\}_{m}\right)\right]^{\alpha / 2}
$$

The partition function $Y_{L}\left(\left\{\mathbf{r}_{m}\right\}\right)$ is the product of statistically independent partition functions $X\left(\mathbf{r}_{j-1}, \mathbf{r}_{j}\right)$ with a factor $\tilde{X}_{L}\left(\mathbf{r}_{m}\right)$. Here $X\left(\mathbf{r}_{j-1}, \mathbf{r}_{j}\right)$ is the partition function for pairs of walks that start at $\mathbf{r}_{j-1}$ and end at their first subsequent intersection $\mathbf{r}_{j}$, whereas $\tilde{X}_{L}\left(\mathbf{r}_{m}\right)$ is the partition function for pairs of walks that start at $\mathbf{r}_{m}$ and do not meet. The length of these walks is $L-r_{m}$, where $r_{m}$ is the number of steps from the origin to $\mathbf{r}_{m}$. Thus, (20) becomes

$$
\left[Z_{L}\right]^{\alpha} \leqslant \sum_{m=0}^{L} \sum_{\{\mathbf{r}\}_{m}}\left[\tilde{X}_{L}\left(\mathbf{r}_{m}\right)\right]^{\alpha / 2} \prod_{j=1}^{m}\left[X\left(\mathbf{r}_{j-1}, \mathbf{r}_{j}\right)\right]^{\alpha / 2}
$$

To perform the averaging, we use the convexity inequality (14). For $\tilde{X}_{L}\left(\mathbf{r}_{m}\right)$ we have

$$
\begin{aligned}
\left\langle\tilde{X}_{L}\left(\mathbf{r}_{m}\right)^{\alpha / 2}\right\rangle & \leqslant\left\langle\tilde{X}_{L}\left(\mathbf{r}_{m}\right)\right\rangle^{\alpha / 2} \\
& =\left[d^{2\left(L-r_{m}\right)} P_{0}\left(L-r_{m}\right)\left\langle e^{-\beta \varepsilon}\right\rangle^{2\left(L-r_{m}\right)}\right]^{\alpha / 2} \\
& =\left\langle Z_{L}\right\rangle^{\alpha} \frac{\left[P_{0}\left(L-r_{m}\right)\right]^{\alpha / 2}}{\left[d\left\langle e^{-\beta \varepsilon}\right\rangle\right]^{\alpha r_{m}}}
\end{aligned}
$$

where $P_{0}(s)$ is the probability that two walks do not meet after $s$ steps [the number of pairs of walks of length $L-r_{m}$ that do not meet is of course $\left.P_{0}\left(L-r_{m}\right) d^{2\left(L-r_{m}\right)}\right]$. For $s \rightarrow \infty, P_{0}(s)$ has a nonzero limit $P_{0}$ in dimension $d>3$. The translational invariance of the lattice means that $\left\langle X\left(\mathbf{r}_{j-1}, \mathbf{r}_{j}\right)\right\rangle$ 
depends on $\mathbf{r}_{j}-\mathbf{r}_{j-1}$. We use this when averaging (21), in the limit of $L \rightarrow \infty$, to interchange the sum over $\{\mathbf{r}\}_{m}$ and product. Then (13), (21), and (22) give

$$
R_{\alpha} \leqslant P_{0}^{\alpha / 2} \sum_{m=0}^{\infty}\left[\sum_{\mathbf{r}} \frac{\left\langle[X(\mathbf{o}, \mathbf{r})]^{\alpha / 2}\right\rangle}{\left(d\left\langle e^{-\beta \varepsilon}\right\rangle\right)^{\alpha r}}\right]^{m}
$$

It finally remains to estimate $\left\langle[X(\mathbf{o}, \mathbf{r})]^{\alpha / 2}\right\rangle$. Now, $X(\mathbf{o}, \mathbf{r})$ is the partition function of pairs of walks which start at $\mathbf{o}$ and meet for the first time at $\mathbf{r}$. There are $d^{2 r} Q_{1}(\mathbf{r})$ such pairs of walks, where $Q_{1}(\mathbf{r})$ is the probability that two directed walks starting from o meet for the first time at site $\mathbf{r}$. The energies of the two walks within a pair are uncorrelated except for the contribution of site $\mathbf{r}$, which is the same for both walks in all pairs. We can factorize this contribution and then use convexity to give

$$
\begin{aligned}
\left\langle[X(\mathbf{o}, \mathbf{r})]^{\alpha / 2}\right\rangle & =\left\langle e^{-\alpha \beta \varepsilon}\right\rangle\left\langle\left[\frac{X(\mathbf{o}, \mathbf{r})}{e^{-2 \beta \varepsilon_{r}}}\right]^{\alpha / 2}\right\rangle \\
& \leqslant\left[Q_{1}(\mathbf{r})\right]^{\alpha / 2} d^{\alpha r}\left\langle e^{-\alpha \beta \varepsilon}\right\rangle\left\langle e^{-\beta \varepsilon}\right\rangle^{\alpha(r-1)}
\end{aligned}
$$

After inserting (24) into (23), we have

$$
R_{\alpha} \leqslant P_{0}^{\alpha / 2} \sum_{m=0}^{\infty}\left\{\frac{\left\langle e^{-\alpha \beta \varepsilon}\right\rangle}{\left\langle e^{-\beta \varepsilon}\right\rangle^{\alpha}} \sum_{\mathbf{r}}\left[Q_{1}(\mathbf{r})\right]^{\alpha / 2}\right\}^{m}
$$

If the rhs of (25) is finite, $R_{x}$ is certainly finite; thus, an upper bound $T_{x}^{u}$ on $T_{\alpha}$ is given when

$$
\frac{\left\langle e^{-\alpha \beta \varepsilon}\right\rangle}{\left\langle e^{-\beta \varepsilon}\right\rangle^{\alpha}} \sum_{\mathbf{r}}\left[Q_{1}(\mathbf{r})\right]^{\alpha / 2}=1 \quad \text { with } \quad \beta=\left[T_{\alpha}^{u}\right]^{-1}
$$

A special case of (25) and (26) is $\alpha=2$. In this case the inequalities (14) and (15) used to derive (25) are equalities and (8) is recovered. The sum in (26) is then the probability of two walks meeting for the first time anywhere. This is equal to the probability that two walks meet at least once, denoted by $A$ in (7).

To check that (25) recovers the result of ref. 11, we again consider polymers on a tree of branching ratio $d$. In this case paths can only meet for the first time at the first step and (25) is easy to calculate:

$$
\sum_{\mathbf{r}}\left[Q_{1}(\mathbf{r})\right]^{\alpha / 2}=d^{(1-\alpha)}
$$


For Gaussian energies, (26) then gives

$$
T_{\alpha}^{u}=\left(\frac{\alpha}{2 \log d}\right)^{1 / 2}
$$

which, as $\alpha \rightarrow 1$, recovers the known transition temperature $T_{c}=T_{0}$.

One should note that we consider here the limit $\alpha \rightarrow 1$ rather than the usual limit $\alpha \rightarrow 0$ of the replica approach. For $\alpha<1$, the inequality (16) is reversed so that $R_{\alpha} \leqslant 1$. It can be shown that when $R_{\alpha}$ is nonzero, the annealed free energy is equal to the quenched free energy. However, upper bounds on $R_{\alpha}$ are not of much use in determining when this occurs.

\section{NUMERICAL RESULTS}

To obtain a value for $T_{\alpha}^{u}(1<\alpha \leqslant 2)$ on the finite-dimensional lattice we first require that for some values of $\alpha<2$

$$
\sum_{\mathbf{r}}\left[Q_{1}(\mathbf{r})\right]^{\alpha / 2}<1
$$

so that (26) has a solution. This is true for $d>3$, since above $d=3$, the probability for two walks to meet is strictly less than one. In addition, to gain an improvement over $T_{2}$ we require

$$
\left.\frac{\partial T_{\alpha}^{u}}{\partial \alpha}\right|_{\alpha=2}>0
$$

The explicit calculation of the sum of probabilities to noninteger powers involved in (25) is difficult and we resoted to their numerical evaluation.

We calculated all the $Q_{1}(\mathbf{r})$ up to eight steps on the lattice by direct enumeration in arbitrary dimension. We also considered the bond version of the problem for which the energies are associated with the bonds of the lattice rather than the sites. In this case we calculated the $Q_{1}(\mathbf{r})$ up to nine steps. In both cases the sum of probabilities converges rather well. One expects this because for $d>3$ the probability of two directed walks ever meeting has a nonzero limit less than one as $L \rightarrow \infty$. This implies that walks are most likely to meet for the first time after a small number of steps.

After each step we use the calculated probabilities to approximate the Ihs of (26). With this curtailed sum we estimated the value of $\alpha$ that through (26) gave the minimum value of $T_{\alpha}^{u}$. The results are shown in Fig. 1, where we plot the minimum over $\alpha$ of $T_{\alpha}^{u}$ against dimension as the number of steps used to calculate $Q_{1}(\mathbf{r})$ increases. At least for large $d$, the 
figure indicates first that the numerical estimation of the minimum $T_{\alpha}^{u}$ does converge and second that the curve to which the results converge gives an improvement over $T_{2}$. The lowest curve on the figure is for $Q_{1}(\mathbf{r})$ calculated up to one step, which recovers the tree [see comment before (27)] and forms a lower bound on $T_{c}$.
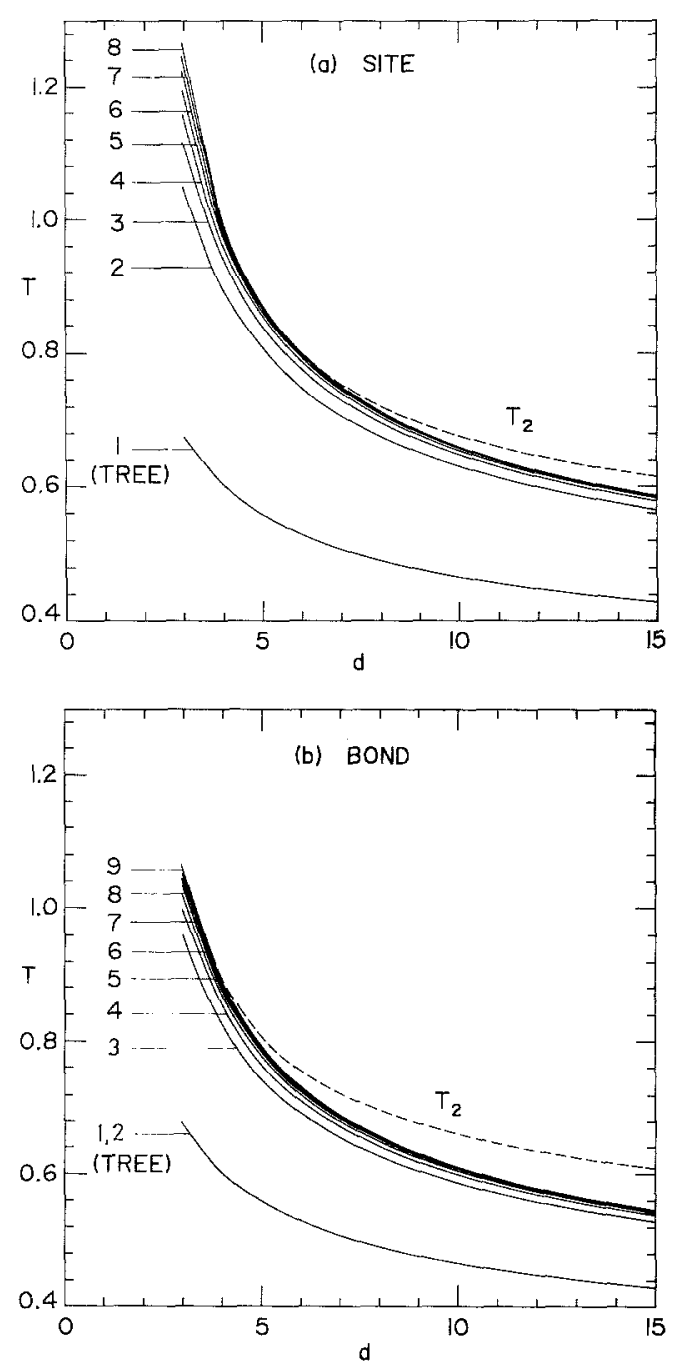

Fig. 1. The full curves plot the upper bound on $T_{c}$ as estimated numerically from (26) for a Gaussian distribution of energies: (a) site energies; (b) bond energies. The number of steps up to which $Q_{1}(\mathbf{r})$ is calculated is marked for each curve. The broken curves are estimates of $T_{2}$ obtained through (26) with $Q_{1}(\mathbf{r})$ calculated up to eight steps for the bond problem and nine steps for the site problem. 
In order to estimate the minimum dimension at which (26) gives an improvement over $T_{2}$, we evaluated the dimension below which (30) no longer holds as the number of steps used to calculate $Q_{1}(\mathbf{r})$ increases. The results are plotted in Fig. 2. The dimensions one could obtain by extrapolating to an infinite number of steps appear different for the bond and site problems, so they probably reflect more the approximations involved in (25) than the dimension at which it is impossible to improve $T_{2}$.

Although we have only considered a Gaussian distribution of energies so far, (25) and (26) are quite general and one could repeat our numerical calculation for other distributions.

We believe that (25) is probably not the optimal bound on $T_{c}$. One way (25) could be improved would be to return to (23) and make more careful estimates on the quantities $\left\langle[X(\mathbf{o}, \mathbf{r})]^{\alpha / 2}\right\rangle$. For example, up to $r=2$ for the site problem and $r=3$ for the bond problem $\left\langle[X(\mathbf{o}, \mathbf{r})]^{\alpha / 2}\right\rangle$ can be calculated exactly. This is because in these cases there is at most one pair of paths that meet for the first time at $\mathbf{r}$. For larger values of $r$ it may be that the use of inequality (15) or a combination of (15) and convexity (14) would give a better estimate of $\left\langle[X(\mathbf{o}, \mathbf{r})]^{\alpha / 2}\right\rangle$ than the method involving convexity alone that we outlined in Section 3 . However, to achieve a significant improvement over (25), one would have to deal with each $\left\langle[X(\mathbf{o}, \mathbf{r})]^{x / 2}\right\rangle$ individually and such a procedure would rapidly become very intricate.

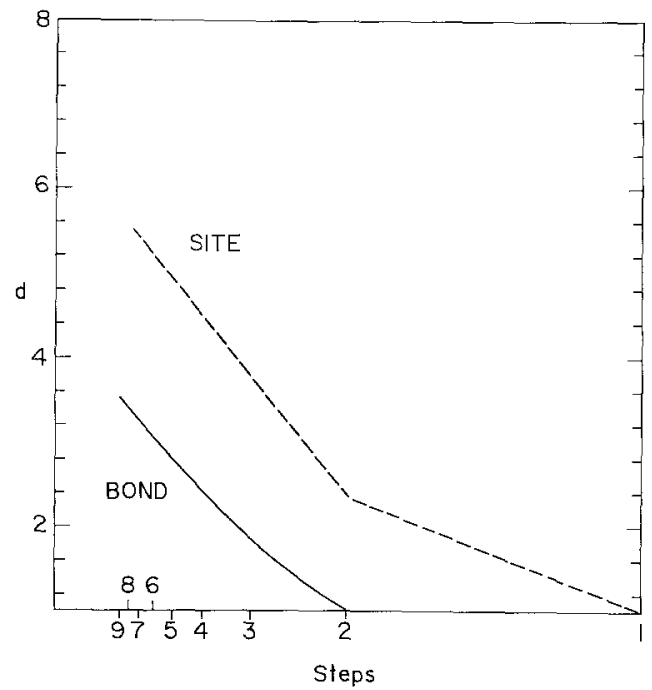

Fig. 2. The minimum dimension at which an improvement over $T_{2}$ is given for Gaussian energies by (26) against the number of steps up to which $Q_{1}(\mathbf{r})$ is calculated to estimate (26). Broken curve, site energies; full curve, bond energies. 
The point we wish to convey in this paper is that for directed polymers in a finite-dimensional random medium one can improve bounds on the transition temperature by a relatively simple method. This method involves estimating noninteger moments of the partition function by the use of wellknown inequalities. First one has to separate the partition function into correlated contributions, then average these contributions as best one can. The basic approach appears rather general and may well be applicable to other strongly disordered systems.

\section{ACKNOWLEDGMENTS}

We would like to thank E. Buffet, B. Chauvin, and A. Rouault for pleasant discussions, the authors of ref. 11 for sending us a copy of their work prior to publication, and E. R. Speer for a critical reading of the manuscript. MRE would like to thank the Royal Society for financial support during this work.

\section{REFERENCES}

1. M. Mézard, G. Parisi, and M. A. Virasoro, Spin Glass Theory and Beyond (World Scientific, Singapore, 1987).

2. B. Derrida and $\dot{H}$. Flyvbjerg, Statistical properties of randomly broken objects and of multivalley structures in disordered systems, J. Phys. A: Math. Gen. 20:5273 (1987).

3. A. Georges, M. Mézard, and J. S. Yedidia, Low-temperature phase of the spin glass on a hypercubic lattice, Phys. Rev. Lett. 64:2937 (1990).

4. Kardar and Y.-C. Zhang, Scaling of directed polymers in a random medium, Phys. Rev. Lett. 58:2087 (1987).

5. J. Krug and H. Spohn, in Solids far from Equilibrium, C. Godrèche, ed. (Cambridge University Press, Cambridge, 1991).

6. B. Derrida and H. Spohn, Polymers on disordered trees, spin glasses and travelling waves, J. Stat. Phys. $51: 817$ (1988).

7. M. Kardar, G. Parisi, and Y.-C. Zhang, Dynamic scaling of growing interfaces, Phys. Rev. Lett. 56:889 (1986).

8. J. Cook and B. Derrida, Directed polymers in a random medium: $1 / d$ expansion and the n-tree approximation, J. Phys. A: Math. Gen. 23:1523 (1990).

9. J. Z. Imbrie and T. J. Spencer, Diffusion of directed polymers in a random environment, J. Stat. Phys. 52:609 (1988).

10. J. Cook and B. Derrida, Polymers on disordered hierarchical lattices: A nonlinear combination of random variables, J. Stat. Phys. 57:89 (1989).

11. E. Buffet, A. Patrick, and J. V. Pulé, Directed polymers on trees: A Martingale approach, Preprint DIAS STP 91-34 (1991).

12. B. Chauvin and A. Rouault, KPP equation and supercritical branching Brownian motion in the subcritical speed area. Application to spatial trees, Prob. Theory Related Fields 80:299 (1988).

13. J. Neveu, Arbres et processus de Galton-Watson, Ann. IHP Prob. Stat. 22:199 (1986).

14. G. H. Hardy, J. E. Littlewood, and G. Pólya, Inequalities (Cambridge University Press, Cambridge, 1934). 